\title{
Jejunal Adenocarcinoma: An Unusual Cause of Small Bowel Obstruction
}

\author{
Boyle $\mathrm{J}^{1}$, Varia $\mathrm{H}^{2}$, Patankar $\mathrm{A}^{3}$ and Pettit $\mathrm{S}^{{ }_{1}}$
}

${ }^{1}$ Departments of General Surgery, Blackpool Teaching Hospitals NHS Foundation Trust, Blackpool, United Kingdom ${ }^{2}$ Departments of Radiology, Blackpool Teaching Hospitals NHS Foundation Trust, Blackpool, United Kingdom ${ }^{3}$ Departments of Histopathology, Blackpool Teaching Hospitals NHS Foundation Trust, Blackpool, United Kingdom

${ }^{*}$ Corresponding author: Pettit S, Consultant Colorectal Surgeon, Department of General Surgery, Blackpool Teaching Hospitals NHS Foundation Trust, Blackpool, United Kingdom, E-mail: stephenhpettit@btinternet. co.uk

Citation: Boyle J, Varia H, Patankar A, Pettit S (2016) Jejunal Adenocarcinoma: An Unusual Cause of Small Bowel Obstruction. J Case Rep Stud 4(5): 501. doi: 10.15744/2348-9820.4.501

Received Date: July 12, 2016 Accepted Date: October 26, 2016 Published Date: October 28, 2016

\begin{abstract}
We report the case of a 52 year old man having no previous abdominal surgery who presented with small bowel obstruction. Computerised tomography showed mid jejunal obstruction, however, the cause of the obstruction could not be diagnosed radiologically. Laparotomy was performed and the obstruction was found to be due to a small annular jejunal tumour. This was resected and histology showed a primary jejunal adenocarcinoma. Staging was T3N1M0. He received adjuvant chemotherapy with Capecitabine and Oxaliplatin and remains disease free two and a half years after surgery.
\end{abstract}

Keywords: Small bowel obstruction; Jejunal adenocarcinoma; Computerised tomography

List of Abbreviations: CT: Computerised tomography; MRI: Magnetic Resonance Imaging

\section{Introduction}

Small bowel obstruction is a frequent surgical emergency. Adhesional obstruction is the commonest cause in patients who have had previous abdominal surgery. In those without previous abdominal surgery common causes of obstruction include obstructed hernias and metastatic malignancy involving the small bowel. Rarer causes include small bowel volvulus, stricturing Crohn's disease, small bowel diaphragm disease caused by non-steroidal anti-inflammatory drug use [1], and small bowel tumours including lymphoma, neuroendocrine tumour, gastrointestinal stromal tumour and small bowel adenocarcinoma. Computerised tomography $(\mathrm{CT})$ will often provide a pre-operative diagnosis to help plan surgical management. Herein we present a case of small bowel obstruction caused by a primary jejunal adenocarcinoma. The details are in the case description below.

\section{Case Report}

A previously fit 52 year old man, having no previous abdominal surgery, presented with a 3 month history of vomiting and 12 kilogram weight loss. There was no succussion splash or abdominal mass on examination. His haemoglobin was normal (136g/L). CT showed small bowel obstruction with the transition point being in the mid-jejunum (Figure 1). There was no tumour mass identifiable on imaging at the point of obstruction.

Laparotomy was performed and the proximal jejunum was found to be chronically obstructed measuring $8 \mathrm{~cm}$ in diameter. The obstruction was caused by a small circumferential tumour in the mid-jejunum (Figure 2) and the distal small bowel was collapsed. The obstructing tumour and adjacent small bowel mesentery were resected en bloc with primary anastomosis. He made an uneventful recovery.

Histology showed a well differentiated adenocarcinoma arising from overlying high grade dysplasia indicating a primary jejunal adenocarcinoma (Figure 3). Focal lymphovascular invasion was present and one of twelve lymph nodes contained metastatic tumour. Staging was T3N1M0. He received 8 cycles of chemotherapy using Capecitabine and Oxaliplatin. Surveillance CT of thorax, abdomen and pelvis at one and two years post-surgery have been normal. He remains well. 


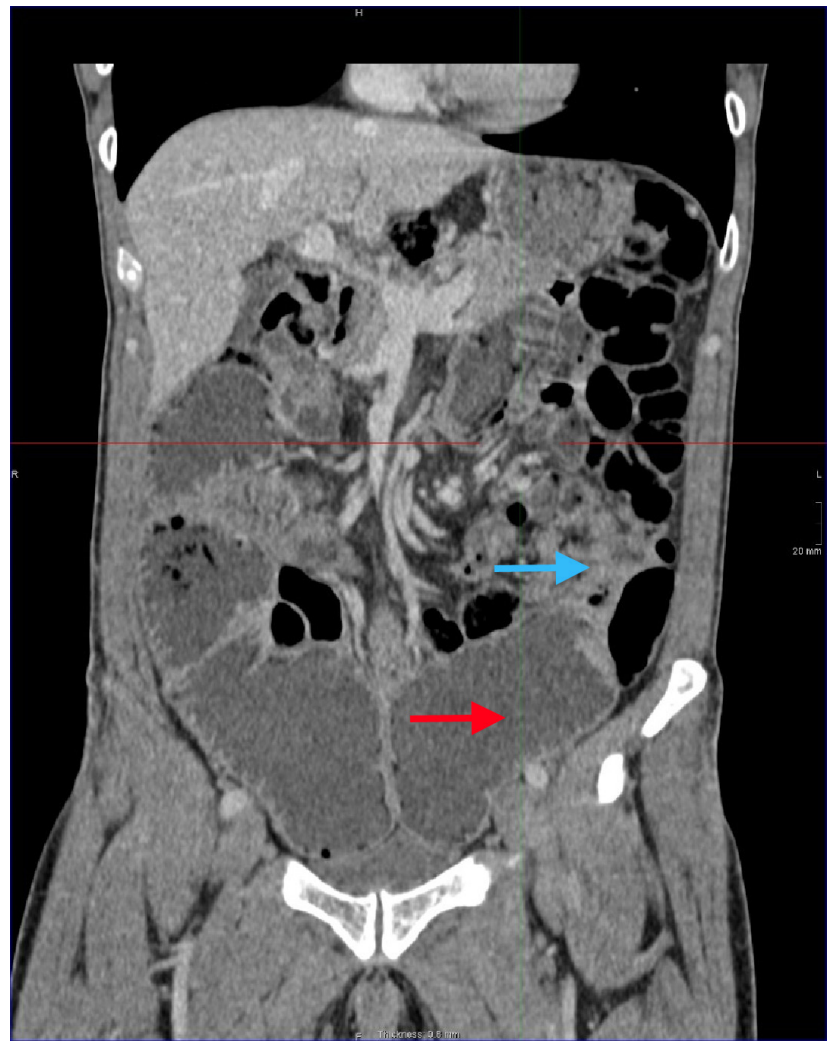

Figure 1: Computerised tomography showing obstructed jejunum (red arrow) and collapsed jejunum (blue arrow)

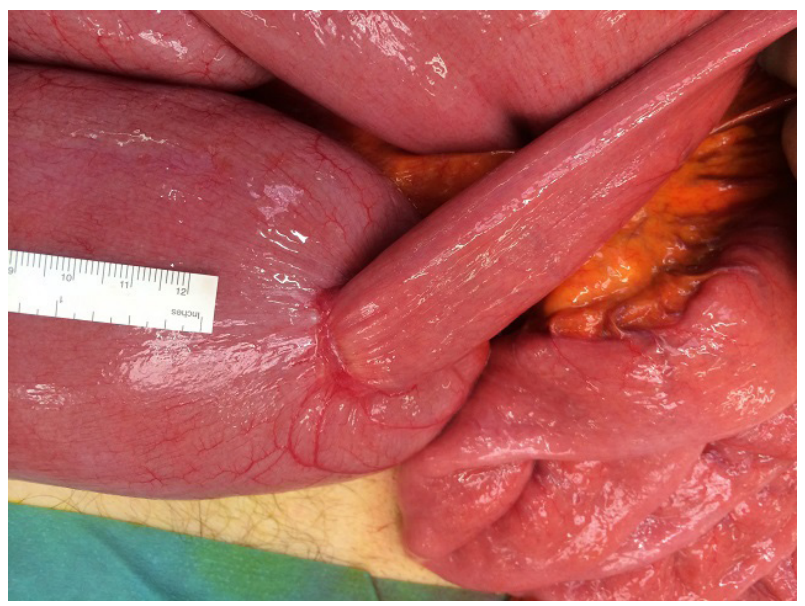

Figure 2: Operative photograph showing the circumferential jejunal adenocarcinoma causing obstruction

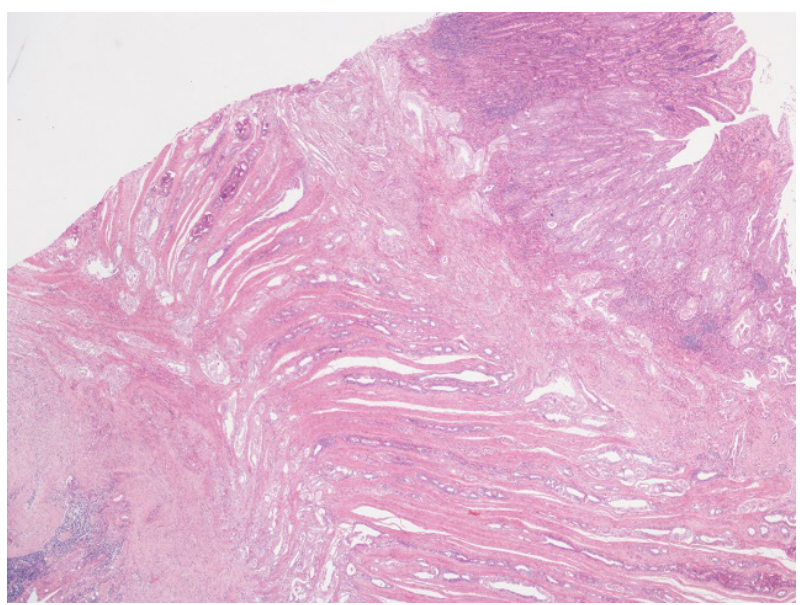

Figure 3: Histopathology slide showing moderately differentiated adenocarcinomas of jejunum diffusely infiltrating the muscle wall and extending into the subserosa focally 


\section{Discussion}

Small bowel adenocarcinoma is rare and accounts for $0.3 \%$ of all malignancies of the gastrointestinal tract [2]. This is because the small bowel is protected from malignant change by factors including rapid transit of small bowel contents, sparseness of bacterial flora and low levels of activating enzymes of pre-carcinogens minimising exposure to potential carcinogenic bacterial breakdown products [3]. There is rapid epithelial cell turnover in the small bowel with an estimated 1 gram of intestinal mucosal cells being lost and replaced every 16 minutes. This rapid turnover prevents malignant change becoming established as cells containing DNA errors are rapidly lost.

Small bowel adenocarcinomas are slightly more common in men than women and the average age at diagnosis is 60 years. Small bowel adenocarcinomas develop from adenomatous polyps that undergo malignant change. There is an increased incidence of small bowel adenocarcinoma in inherited conditions where multiple polyps develop in both the large and small bowel including familial adenomatous polyposis, hereditary non-polyposis colorectal cancer (Lynch syndrome) and MUTYH associated polyposis. There is also an increased incidence of small bowel adenocarcinoma in patients with coeliac disease, small bowel Crohn's disease, Peutz-Jehgers syndrome and cystic fibrosis. Small bowel adenocarcinomas are cytokeratin 20 positive, CDX-2 positive and over $50 \%$ are cytokeratin 7 positive.

In their early stages small bowel adenocarcinomas are asymptomatic and the only sign of their development may be microcytic anaemia. In patients with microcytic anaemia, in the absence of an obvious cause such as menorrhagia in pre-menopausal women, full gastrointestinal investigation is mandatory. Initially gastroscopy with duodenal biopsy and colonoscopy should be performed to exclude conditions causing anaemia such as peptic ulceration, gastric neoplasia, coeliac disease, colonic polyps and colonic neoplasia. If these investigations are normal then small bowel imaging should be performed.

Both CT enterography using thin section multidetector row CT with 3D multiplanar reformation, and magnetic resonance imaging (MRI) enterography using accelerated image acquisition plus breath holding techniques provide diagnostic information about the small bowel lumen and depict mural and extramural pathology. MRI has the advantage of obviating radiation exposure and this is the preferred investigation in children and young adults. To appropriately assess the small bowel distension is essential. This is achieved by administration of isosmotic oral contrast. MRI enterography also has the advantage of providing not only static but functional information, as repetitive single breath hold scanning is applied during the filling process. Morphologically, small bowel tumours can appear as well defined nodular mural lesions, or as a diffusely infiltrating mass with spiculated borders, or as a diffuse or annular wall thickening causing concentric narrowing. On T1 weighted imaging the tumour may be homogenously iso or hypointense, and slightly hyper or isointense on T2 weighted imaging. Contrast enhancement is moderate and mostly homogenous. If cross-sectional imaging is normal then capsule endoscopy and isotope Meckel's scan should be performed. If any imaging technique demonstrates proximal small bowel pathology then biopsy may be possible using double balloon enteroscopy $[4]$.

Many cases of jejunal adenocarcinomas are not diagnosed until they are large enough to cause sub-acute small bowel obstruction, weight loss and severe anaemia. The majority are diagnosed at laparotomy for small bowel obstruction [5]. Due to late presentation many small bowel adenocarcinomas have metastasised by the time of diagnosis giving a poor prognosis [2]. Prognostic data for patients diagnosed with small bowel adenocarcinomas between 1998 and 2002 are given in Table 1 [6], although outcomes may now be improved because of the increasing use of neoadjuvant chemotherapy.

\begin{tabular}{|c|c|c|}
\hline Stage & TNM & 5 Year Survival \\
\hline I & T1 or T2 N0M0 & $55 \%$ \\
\hline IIA & T3N0M0 & $49 \%$ \\
\hline IIB & T4N0M0 & $35 \%$ \\
\hline IIIA & Any T N1M0 & $31 \%$ \\
\hline IIIB & Any T N2M0 & $18 \%$ \\
\hline IV & Any T Any N M1 & $5 \%$ \\
\hline
\end{tabular}

Table 1: Prognosis of patients with small bowel adenocarcinoma according to stage at diagnosis

CT should ideally be performed in all cases of small bowel obstruction as it will give valuable information for surgical planning. The tomograms may suggest a benign cause for obstruction such as adhesions or volvulus, or they may show an obstructing lesion such as Crohn's disease or a small bowel tumour. CT is particularly valuable when an inoperable situation is found such as multiple sites of small bowel obstruction caused by metastatic ovarian carcinoma. Laparotomy should be performed in patients who are medically fit for surgery where CT shows an operable cause for small bowel obstruction. If a small bowel tumour is found at laparotomy then en bloc resection of the tumour and adjacent small bowel mesentery should be performed to remove the field of lymphatic drainage. If histology shows an adenocarcinoma with adverse prognostic factors such as lymphovascular invasion or lymph node metastases then the prognosis may be improved with adjuvant chemotherapy [7]. 


\section{Conclusion}

Diagnostic laparotomy remains the optimal management of patients presenting with small bowel obstruction where CT has shown a potentially operable cause. This allows resolution of the obstruction and provides a definitive diagnosis. If a small bowel tumour is found then resection of the tumour and mesenteric lymphadenectomy is appropriate management.

\section{References}

1. Sarantitis I, Gerrard AD, Teasdale R, Pettit S (2015) Small bowel diaphragm disease mimicking malignancy. BMJ Case Rep 10.1136/bcr-2015-210174.

2. Ugurlu MM, Asoglu O, Potter DD, Barnes SA, Harmsen WS, et al. (2005) Adenocarcinoma of the jejunum and ileum: a 25-year experience. J Gastrointest Surg 9: $1182-8$.

3. Neugut AI, Jacobson JS, Suh S, Mukherjee R, Arber N (1998) The epidemiology of cancer of the small bowel. Cancer Epidemiol Biomarkers Prev 7: 243-51. 4. Takeuchi N, Aoki R, Semba S, Naba K, Maeda T, et al. (2012) Jejunal carcinoma successfully detected by capsule endoscopy and balloon endoscopy: a case report. J Clin Case Rep 10.4172/2165-7920.1000229.

5. Neugut AI, Marvin MR, Rella VA, Chabot JA (1997) An overview of adenocarcinomas of the small intestine. Oncology 11: 529-50.

6. American Joint Committee on Cancer. Small Intestine (2010) In: AJCC Cancer Staging Manual (7 ${ }^{\text {th }}$ Edn) Springer, New York, USA.

7. Chen CW, Wang WM, Su YC, Wu JY, Hsieh JS, et al.(2008) Oxiplatin/5-fluorouracil/leucovorin (FOLFOX 4) regimen as an adjuvant chemotherapy in the treatment of advanced jejunal adenocarcinomas: a report of 2 cases. Med Princ Pract 17: 496-9.

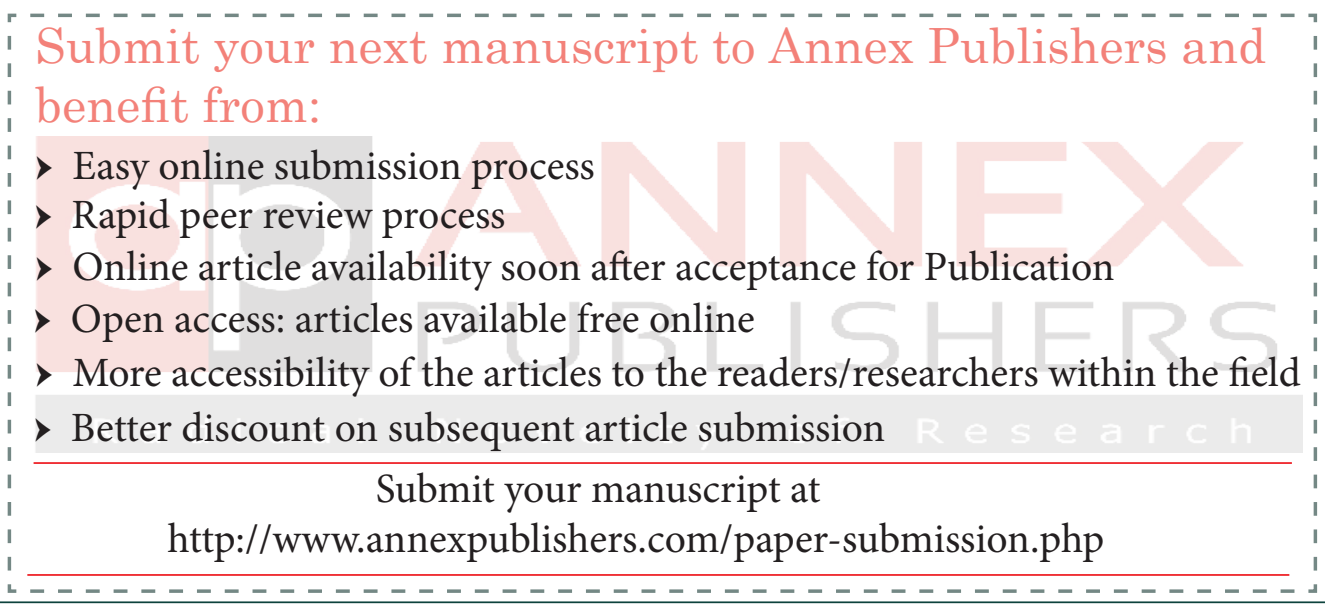

